\title{
Vitamin D and VDR in cancer cachexia and muscle regeneration
}

\author{
Andrea Camperi ${ }^{1,5, *}$, Fabrizio Pin ${ }^{1,2, *}$, Domiziana Costamagna ${ }^{1,2,8}$, Fabio Penna ${ }^{1,2}$, \\ Maria Lopez Menduina ${ }^{1,3}$, Zaira Aversa ${ }^{4}$, Teresa Zimmers ${ }^{5}$, Roberto Verzaro ${ }^{6}$, \\ Raffaella Fittipaldi ${ }^{7}$, Giuseppina Caretti ${ }^{7}$, Francesco Maria Baccino ${ }^{1}$, Maurizio \\ Muscaritoli ${ }^{4}$, Paola Costelli ${ }^{1,2}$ \\ ${ }^{1}$ Department of Clinical and Biological Sciences, University of Turin, Turin, Italy \\ ${ }^{2}$ Interuniversity Institute of Myology, Italy \\ ${ }^{3}$ Department of Physiology, Complutense University of Madrid, Madrid, Spain \\ ${ }^{4}$ Department of Clinical Medicine, Sapienza University of Rome, Italy \\ ${ }^{5}$ Indiana University School of Medicine - IUPUI, Indianapolis, IN, USA \\ ${ }^{6}$ Department of Surgery, M.G. Vannini Hospital, Rome, Italy \\ ${ }^{7}$ Department of Biosciences, University of Milan, Milan, Italy \\ ${ }^{8}$ Current address: Translational Cardiomyology Laboratory, Stem Cell Biology and Embryology, Department of Development \\ and Regeneration, University Hospital Gasthuisberg, Leuven, Belgium \\ *Andrea Camperi and Fabrizio Pin equally contributed to the present study \\ Correspondence to: Paola Costelli, email: paola.costelli@unito.it \\ Keywords: muscle wasting, regeneration, vitamin D receptor, myogenin, circulating vitamin D
}

Received: July 28, $2016 \quad$ Accepted: January 27, $2017 \quad$ Published: February 21, 2017

\section{ABSTRACT}

Low circulating levels of vitamin D were associated with decreased muscle strength and physical performance. Along this line, the present study was aimed to investigate: i) the therapeutic potential of vitamin $D$ in cancer-induced muscle wasting; $\mathrm{i}$ ) the mechanisms by which vitamin $\mathrm{D}$ affects muscle phenotype in tumorbearing animals.

Rats bearing the AH130 hepatoma showed decreased circulating vitamin D compared to control rats, while muscle vitamin D receptor (VDR) mRNA was upregulated. Both circulating vitamin $D$ and muscle VDR expression increased after vitamin $D$ administration, without exerting appreciable effects on body weight and muscle mass.

The effects of vitamin D on muscle cells were studied in C2C12 myocytes. Vitamin D-treated myoblasts did not differentiate properly, fusing only partially and forming multinucleated structures with aberrant shape and low myosin heavy chain content. Vitamin D treatment resulted in VDR overexpression and myogenin down-regulation. Silencing VDR expression in C2C12 cultures abrogated the inhibition of differentiation exerted by vitamin $D$ treatment.

These data suggest that VDR overexpression in tumor-bearing animals contributes to muscle wasting by impairing muscle regenerative program. In this regard, attention should be paid when considering vitamin $D$ supplementation to patients affected by chronic pathologies where muscle regeneration may be involved.

\section{INTRODUCTION}

Cachexia is a comorbidity of cancer [1] characterized by progressive loss of body weight as well as of skeletal muscle and adipose tissue mass. More than $50 \%$ of all cancer patients develop cachexia. This percentage increases up to $86 \%$ during the last 2 weeks of life and about $20 \%$ of all cancer deaths can be attributable to cachexia. Moreover, cachexia impairs quality of life, as well as tolerance and response to anti-neoplastic treatments (reviewed in [2]).

Anorexia, inflammation and perturbations of hormonal and metabolic homeostasis significantly contribute to the onset and progression of cachexia. In this 
regard insulin, angiotensin, leptin and myostatin, together with numerous cytokines, including IL6, IL1 and TNF $\alpha$ have been shown to play a role $[2,3]$.

Skeletal muscle wasting is among the most important cilnical features of cancer cachexia. It mainly derives from the activation of a protein hypercatabolic response that involves different proteolytic systems, in particular the ubiquitin-proteasome-dependent pathway [4] and autophagy However, other mechanisms have also been suggested to contribute to muscle depletion, such as down-regulation of protein synthesis (see [2]) and impaired myogenic response $[5,6]$.

Vitamin (Vit) D3, the precursor of biologically active VitD, is a pleiotropic hormone synthesized mainly in the skin via a UV-dependent reaction. VitD3 is then transported to the liver where it is hydroxylated at the $\mathrm{C}-25$ position to produce $25(\mathrm{OH})-\mathrm{VitD}$, the major circulating form of this hormone. In order to become active, 25(OH)-VitD must be hydroxylated in the C-1 position, producing $1,25(\mathrm{OH})$-VitD (hereafter referred to as VitD). This enzymatic reaction takes place mainly in the kidney, although other cells/tissues express the CYP27BI enzyme required for hydroxylation [7]. VitD circulates in the blood bound to vitamin D-binding proteins, reaching its target tissues to exert endocrine actions. These latter are mediated by the vitamin D receptor (VDR), a member of the nuclear receptor family of transcription factors, which is expressed in different tissues (reviewed in [8]). VDR knock-out mice show the full phenotype of severe vitamin $\mathrm{D}$ deficiency, indicating that VDR is the major mediator of VitD action [9]. Upon binding to VitD, VDR forms a heterodimer with the retinoid-X receptor and regulates the expression of genes whose promoters contain specific DNA sequences known as VDR responsive elements (VDRE). A number of coactivators and corepressors are involved in this transcriptional regulation, providing context, tissue and target gene specificity [8]. However, some actions of VitD have been shown to be more immediate than those described above, and have been suggested to depend on engagement of a membrane associated VDR [10].

The classical and best known functions of VitD involve the regulation of calcium and phosphate metabolism, mainly acting on bone tissue, intestine and kidneys [11]. More recently, VitD has been demonstrated to play a role in several physiological and pathological processes, such as immune response and cancer development [8].

VitD deficiency has been shown to cause abnormalities in skeletal muscle, such as reduced actomyosin content, decrease in mitochondrial $\mathrm{Ca}^{2+}$ levels, reduced $\mathrm{Ca}^{2+}$ uptake in the sarcoplasmic reticulum and low creatine kinase serum levels [12]. VitD deficiency is common in aged individuals, and has been proposed as an independent indicator of frailty and mortality [13]. Recently, VitD deficiency has been shown to result in muscle wasting with increased protein breakdown due to hyperactivation of the ubiquitin-proteasome pathway [14]. Consistent with a protective role of VitD in muscle, VitD supplementation in elderly patients has been shown to improve skeletal muscle functions, such as increased lower limb muscle strength [15] and reduction in the relative risk of falls [16]. Nevertheless, a number of studies have failed to demonstrate an effect of VitD on physical performance $[17,18]$, a matter that is still controversial [19].

VitD effects on muscle cells are long known [20], and several observational studies have been performed on the role of VitD on skeletal muscle. VDR knock-out mice have provided evidences for a direct role of this steroid receptor in the skeletal muscle. Endo and coworkers (2003) have demonstrated that myofibers in VDR-null mutant mice are smaller than in wild type mice. Moreover, they showed that skeletal muscles from 3 weeks old mice lacking VDR display an abnormally elevated expression of myogenic factors such as Myogenin, Myf5 and E2A, as well as increased expression of embryonic and neonatal myosin heavy chain (MyHC; [21]). Taken together, these data suggest that VDR is an important regulator of skeletal muscle development and differentiation.

Despite evidence demonstrating that VitD acts on the skeletal muscle, the effectiveness of VitD administration in pathological conditions characterized by muscle atrophy has not been assessed. For this reason, the present study was designed to investigate: i) the role of VitD in the pathogenesis of cancer cachexia; ii) the possibility to use VitD treatment to prevent muscle wasting in an experimental model of cancer cachexia (rats bearing the AH130 Yoshida ascites hepatoma); iii) the mechanisms by which vitamin D might affect muscle phenotype in tumor-bearing animals. The results obtained show that, while VitD does not significantly improve cachexia, it does affect myogenesis.

\section{RESULTS}

\section{VitD administration did not prevent cachexia in the AH130 hosts}

Rats bearing the AH130 hepatoma represent a well characterized model for the study of cancer cachexia, showing a progressive loss of body weight that parallels tumor growth [22]. Body weight loss in the AH130 bearers is associated with severe depletion of skeletal muscle mass (Figure 1A) and with significant reduction of VitD plasma levels below control values (Figure 1B). Low circulating VitD, is paralleled by a significant increase of VDR mRNA in the skeletal muscle of the AH130 hosts (Figure 1C).

The administration of VitD3 (80 IU $/ \mathrm{kg}$, daily intragastrical administration, starting the day of transplantation) increases $25(\mathrm{OH})$-VitD circulating levels (about 35\% above baseline) in both controls and AH130 hosts (Figure 1B). However, no appreciable effects on 
tumor mass (data not shown), final body weight (FBW), gastrocnemius and tibialis muscle mass (GSN and TIB respectively) can be observed in treated $v s$ untreated animals (Figure 1A). Consistent with previous in vitro and in vivo observations [23], VitD3 supplementation induced an increase above baseline of VDR mRNA expression in the tibialis muscle of control animals (confirming data by Girgis et al [24]) and further exacerbated the increase occurring in the AH130 hosts (Figure 1C).

\section{Muscle VDR overexpression was observed in tumor hosts}

To ascertain that VDR overexpression in the skeletal muscle is not a peculiar feature of the AH130-bearing rats,
VDR levels were assessed also in mice transplanted with the Lewis lung carcinoma (LLC) or with the Colon 26 carcinoma (C26), both being able to induce cachexia in the host mouse $[6,25]$.

Mice injected with LLC cells show a significant decrease in body weight and skeletal muscle mass, as reported in Figure 2A. Although 25(OH)-VitD plasma levels were not significantly different between controls and tumor-bearing mice (Figure 2B), VDR protein expression was increased in the skeletal muscle of the latter (Figure 2B, 2C).

C26 tumor growth is associated with a marked loss of body weight, as well as of both gastrocnemius and tibialis muscle mass (Figure 2D). Both 25(OH)-VitD levels and VDR expression were increased in the C26
A

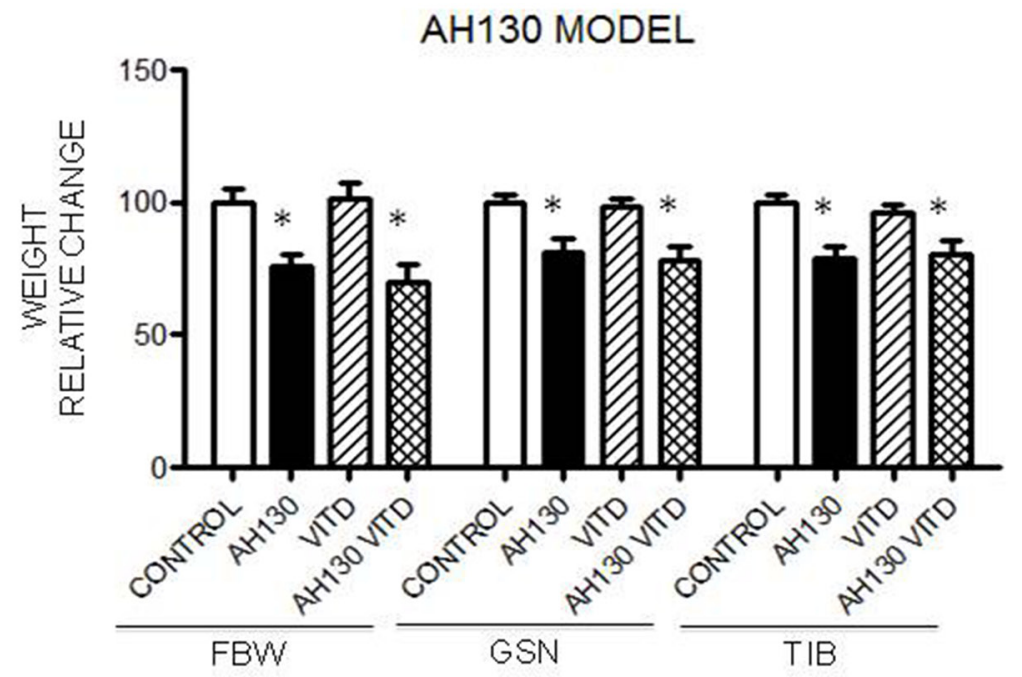

B

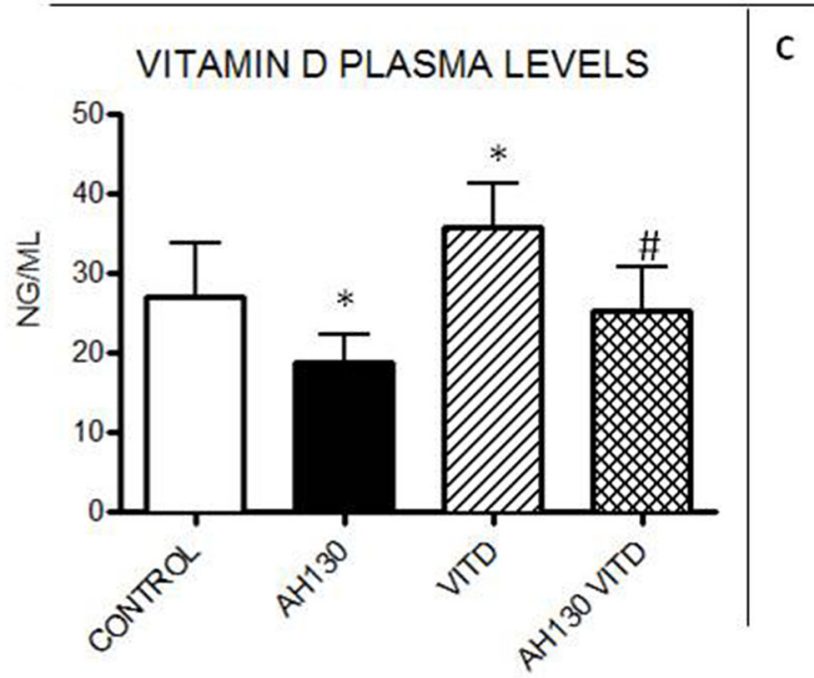

C

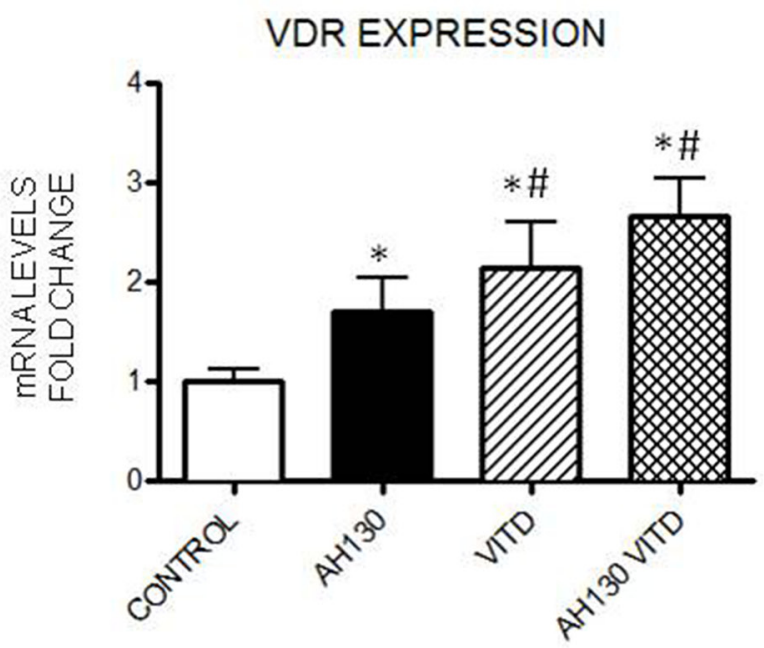

Figure 1: Effects of VitD3 administration to rats bearing the AH130 hepatoma. A. Body weight changes, gastrocnemius and tibialis muscle weight in controls and tumor-bearing rats either untreated or receiving VitD3. B. VitD plasma levels in untreated and VitD3-treated rats. C. VDR mRNA expression levels in the tibialis muscle of untreated and VitD3-treated animals. Data (means \pm SEM) are expressed as fold change (control rats: $\mathrm{n}=6$, tumor-bearing rats: $\mathrm{n}=8$ ). Significance of the differences: ${ }^{*} \mathrm{p}<0.05 v s$ control; $\# \mathrm{p}<0.05 v s$ AH130. 
bearers with respect to control mice (Figures 2E, 2F). $25(\mathrm{OH})-\mathrm{VitD}$ circulating levels were markedly lower in control Balb/c mice than in control C57BL/6J animals, conforming to previous data [26]. The administration of VitD3 to the C26 hosts produced results comparable to those reported in rats bearing the AH-130 hepatoma (Supplementary Figure 1).

Finally, VDR mRNA expression was also increased in skeletal muscle biopsies of cancer patients with respect to those of control subjects (Supplementary Figure 2).

\section{Muscle VDR levels regulate myogenic differentiation}

The VDR signaling pathway has been linked to defects in skeletal muscle development [21]. To investigate this point, the effects of VitD treatment were studied in vitro on $\mathrm{C} 2 \mathrm{C} 12$ myocyte cultures. VitD addition to the culture medium slowed $\mathrm{C} 2 \mathrm{C} 12$ myoblast proliferation in a dose- and VDR-dependent manner (data not shown), confirming previous observations [24, 27]. Moreover, attempts to produce VDR overexpressing $\mathrm{C} 2 \mathrm{C} 12$ myoblasts by gene transfection were revealed to be unsuccessful, suggesting that high VDR levels might be toxic, in myoblasts at least, lowering proliferation rates and eventually leading to cell death (data not shown).

To test if VitD could affect myogenic differentiation, C2C12 myoblasts were grown to confluence and then induced to differentiate in the appropriate medium, with or without $10 \mathrm{nM} 1.25-\mathrm{OH}^{2}$-vitD for 4 days. As demonstrated by immunofluorescence for MyHC (Figure $3 \mathrm{~A})$, control myoblasts were able to fuse together and to form myotubes with the canonical elongated structure, while VitD treated cells only partially fused (avarage number of nuclei/myotube: $C=6.26 \pm 1.99$, $\mathrm{VitD}=3.33 \pm 1.56, \mathrm{p}<0.05, \mathrm{n}=200$. Total number of nuclei:
A

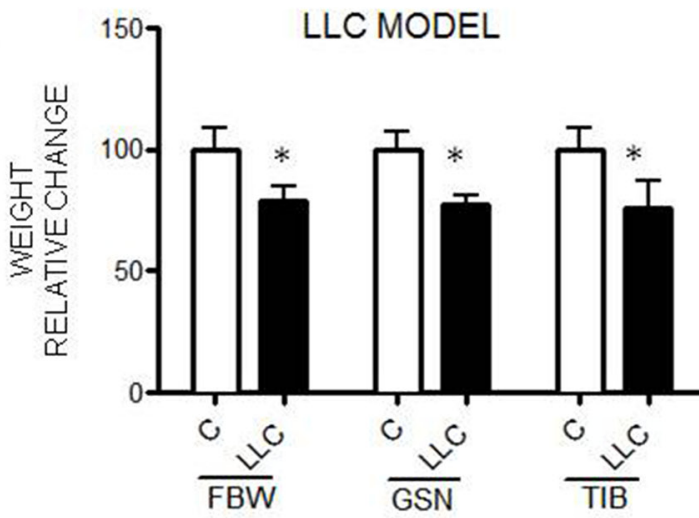

B
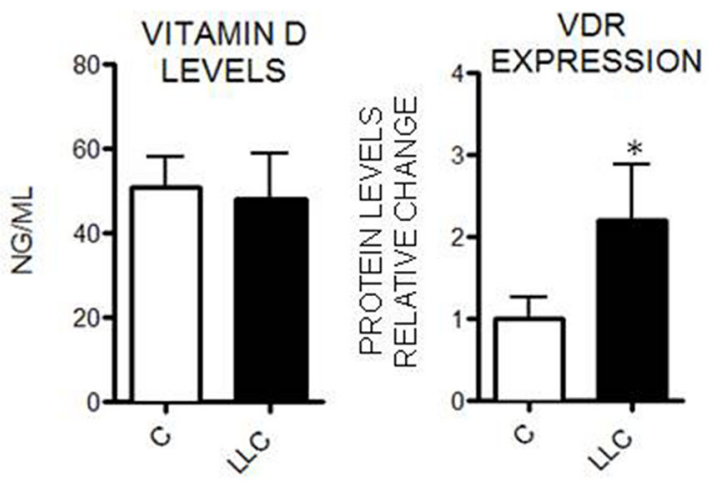

C

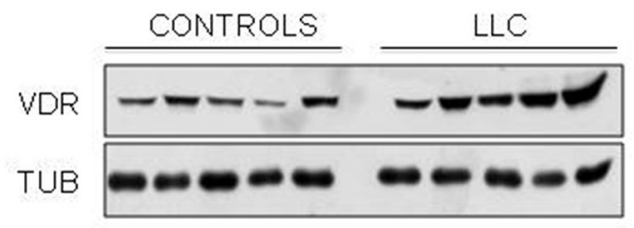

D

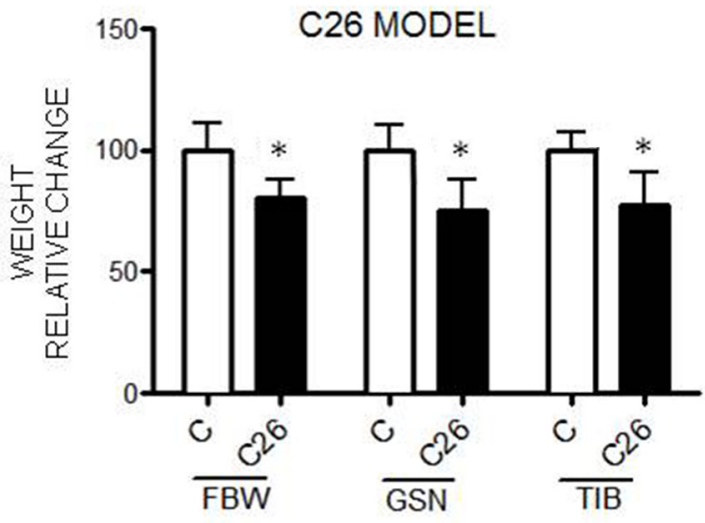

E

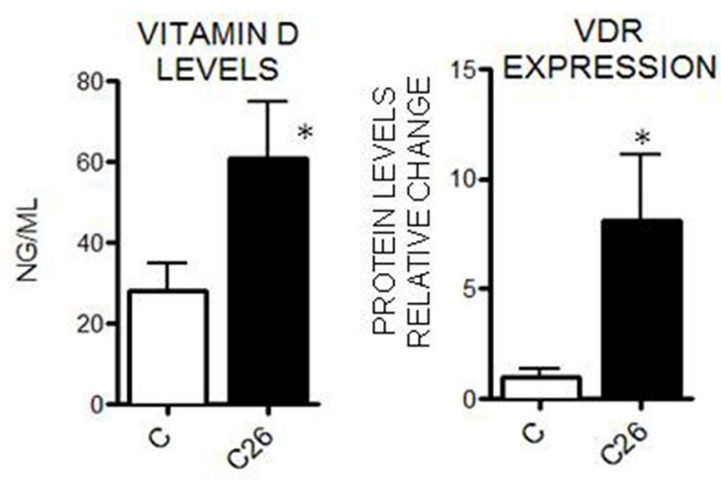

$\mathbf{F}$

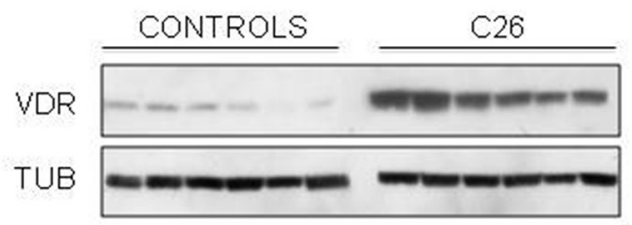

Figure 2: VitD levels and VDR expression in mice bearing the LLC or the C26 tumor. A, D. Final body weight (FBW), gastrocnemius (GSN) and tibialis (TIB) mass in controls $(n=5)$ and tumor-bearing mice $(n=7)$. B, E. Circulating VitD and VDR protein expression levels in GSN muscle. C, F. Representative WB analysis of VDR expression in the gastrocnemius (anti-VDR antibody, clone D6, Santa Cruz Biotechnology). Data are means \pm SD. Significance of the differences: ${ }^{*} \mathrm{p}<0.05$ vs $\mathrm{C}$. 
$\mathrm{C}=83.66 \pm 13.01, \mathrm{Vit} \mathrm{D}=58.66 \pm 14.67, \mathrm{p}=0.09, \mathrm{n}=3)$ and give rise to multinucleated structures with aberrant shape and low MyHC content (Figure 3A and Supplementary Figure 3A). Western blotting analysis showed a rapid decline of VDR levels as differentiation progressed; such reduction is paralleled by increased levels of myogenin, one of the markers of myogenic differentiation (Figure 3B, Supplementary Figure 3B). When differentiation was induced in the presence of VitD, the levels of myogenic regulatory factors such as Pax 7 and myogenin were significantly reduced, at both 2 and 4 days after shifting to differentiation medium, while the expression of MyoD was not different from controls (Figure 3C, Supplementary Figure 3C). At the same time, in parallel to myogenin reduction, VDR was overexpressed at both mRNA (Supplementary Figure 3D) and protein level (Figure $4 \mathrm{~A})$, in a negatively correlated manner $\left(\mathrm{r}^{2}=0.89\right.$, p-value $=0.0031)$.

To ascertain if VitD treatment could also affect mature myotubes, $\mathrm{C} 2 \mathrm{C} 12$ myoblasts were differentiated for 4 days in regular differentiation medium and then treated with for $48 \mathrm{~h}$ with VitD (10-100 $\mathrm{nM})$ or with IL-6, as a positive control of fiber shrinkage [28]. The results show that only $100 \mathrm{nM}$ VitD significantly decreased myotube size (Figure 4B), while the number of nuclei per myotube was significantly reduced by both VitD concentrations (Figure 4B). Interestingly, although IL-6 treatment was comparable to $100 \mathrm{nM}$ VitD as for myotube shrinkage, it did not affect the number of nuclei per myotube (Figure 4B), suggesting that two different mechanisms are responsible for VitD and IL-6-induced decrease of myotube size. Finally, VitD, but not IL-6, treatment of $\mathrm{C} 2 \mathrm{C} 12$ myotubes markedly increased VDR expression (Figure 4C).

The observation reported above that myogenin and VDR expression are negatively correlated suggested the occurrence of a direct transcriptional regulator activity of VDR. To investigate this point, the promoter region of the mouse myogenin gene was analyzed in silico for the presence of putative VDRE. Figure 5A shows the
A

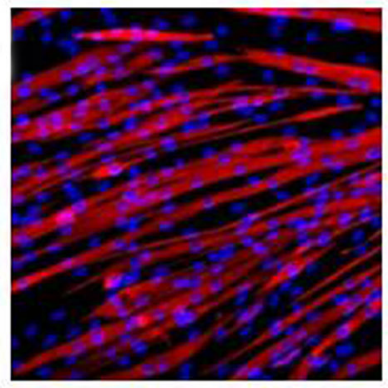

C

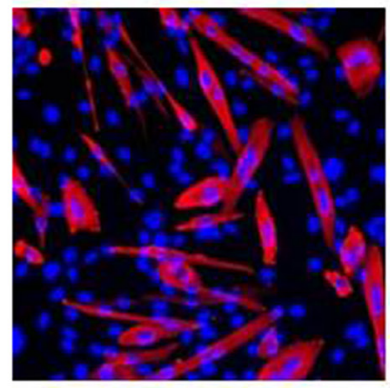

VIT D 10nM

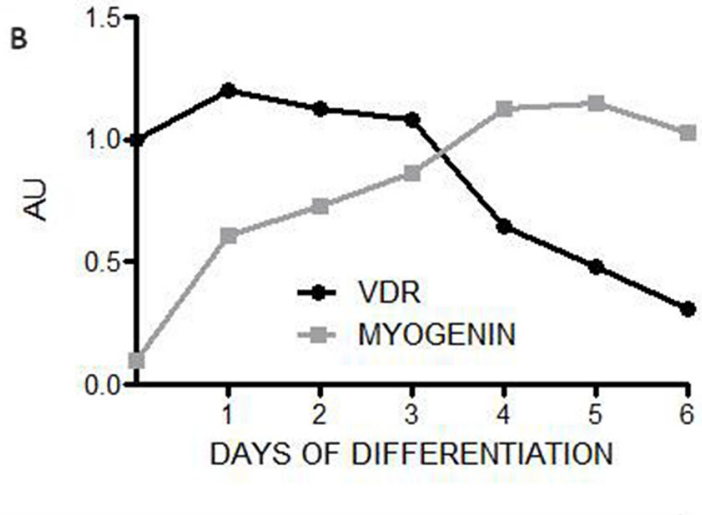

C

PAX7

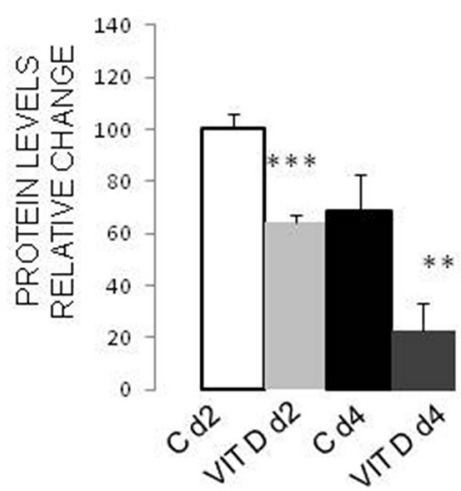

MYOGENIN

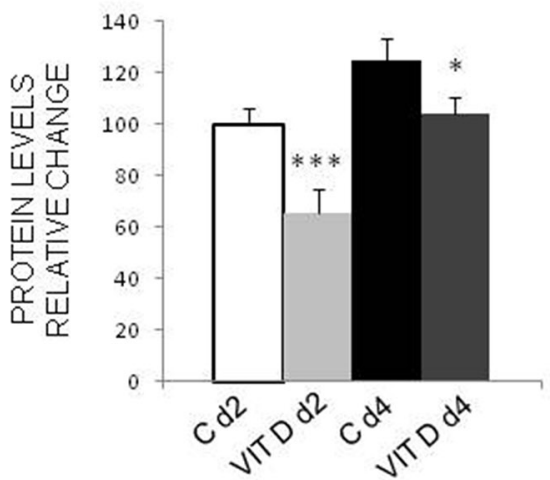

MyoD

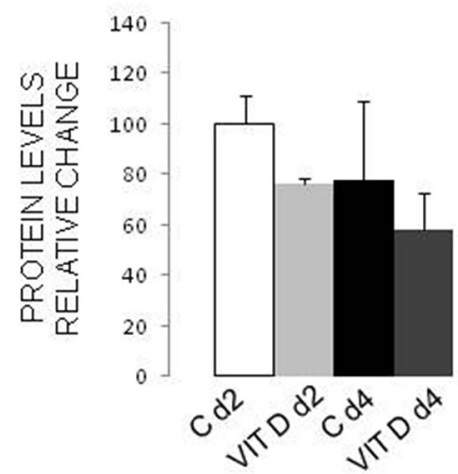

Figure 3: Effects of VitD on C2C12 myoblast differentiation. A. Immunostaining of control or VitD-treated C2C12 cells after 4 days of differentiation (Red: MyHC, Blue: nuclei). B. Expression levels of VDR (black line) and myogenin (grey line) during C2C12 myoblast differentiation in the absence of VitD treatment, as measured by western blotting analysis (Santa Cruz Biotechnology: anti-VDR antibody, clone D6; anti-myogenin antibody, clone F5D). C. Protein expression levels of some myogenic regulatory factors in control or VitD-stimulated C2C12 cells at day 2 and 4 of differentiation (Santa Cruz Biotechnology: anti-myogenin antibody, clone F5D, anti-MyoD antibody, clone M318; Developmental Studies Hybridoma Bank, University of Iowa: anti-Pax7 antibody). Data (means \pm SD) are expressed as $\%$ of controls. Significance of the differences: $* * * p<0.001, * * p<0.01,{ }^{*} p<0.05$ vs $\mathrm{C}, 3$ independent experiments. 
presence of 15 predicted VDR responsive elements in the $10 \mathrm{~kb}$ upstream myogenin transcription start, six of which placed in the last $5 \mathrm{~kb}$ upstream the coding region. Chromatin immunoprecipitation was performed to validate the in silico analysis. The results demonstrate that three among the putative binding sites exhibited an enrichment after immunoprecipitation with anti-VDR antibody, demonstrating a direct interaction of VDR with the myogenin promoter (Probes 1, 3, 7; Figure 5B).

\section{VDR silencing restores $\mathrm{C} 2 \mathrm{C} 12$ myoblast differentiation}

To test the relevance of VDR to myogenesis, its expression was silenced by means of lentivirus-delivered specific shRNA. In this condition (shVDR), C2C12 cells were no longer sensitive to VitD treatment and differentiated normally into mature myotubes (Figure 6A), as also indicated by the number of nuclei/myotube (Figure $6 \mathrm{C})$. Figure $6 \mathrm{~B}$ shows that VDR silencing was robust $(-83 \%$ in shVDR cells compared to shC) and that the marked reduction of both myogenin and $\mathrm{MyHC}$ expression induced by VitD treatment in ShC infected cells was not detectable in shVDR cultures (Figure 6C). Intriguingly, untreated ShVDR cultures expressed more myogenin and MyHC than untreated ShC cells (Figure 6B, 6C), suggesting that the lack of VDR enhanced myogenic differentiation even in basal conditions. When shVDR cells were stimulated with $10 \mathrm{nM}$ VitD, the expression levels of both VDR and myogenin were at least as high as in ShC untreated cells.

\section{VitD administration impairs muscle regeneration in vivo}

To test whether the anti-differentiation effects mediated by VitD and VDR in vitro occur also in vivo,

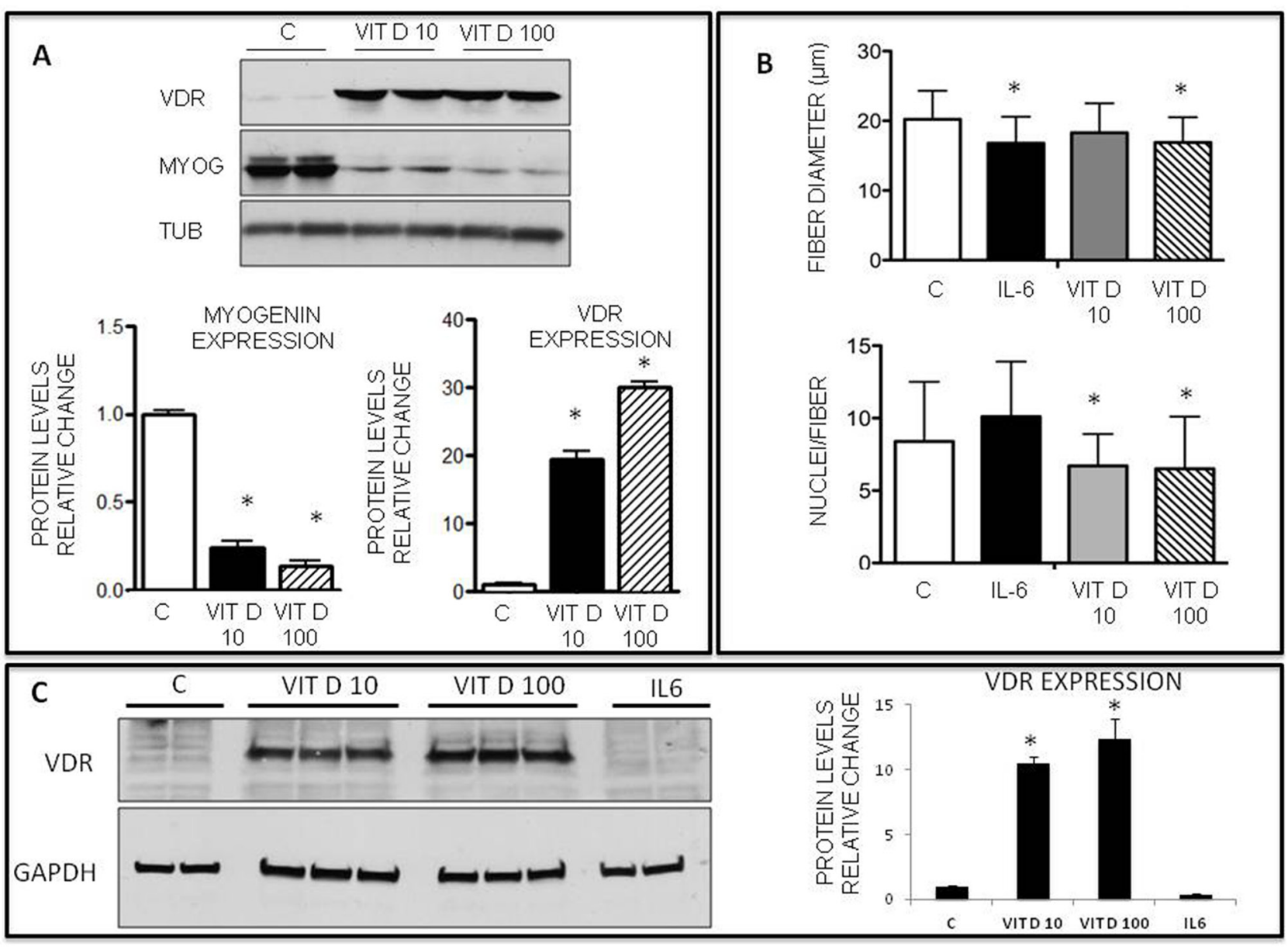

Figure 4: Effects of VitD on VDR and myogenin expression on C2C12 differentiating myoblasts and on myotubes. A. Protein expression levels of VDR and myogenin in control or VitD-stimulated C2C12 cells at day 4 of differentiation (Santa Cruz Biotechnology: anti-VDR antibody, clone D6, anti-myogenin antibody, clone F5D); B. Average myotube diameter and average number of nuclei/myotube in $\mathrm{C} 2 \mathrm{C} 12$ cultures treated with control medium or with medium containing: 100ng/ml IL6, 10nM or 100nM VitD (bars show the average of three independent experiments, $n=100$ for each condition); C. Western blotting analysis of representative samples showing VDR expression (Santa Cruz Biotechnology: anti-VDR antibody, clone D6). Data are means \pm SD. Significance of the differences: $* \mathrm{p}<0.05$ vs C, 3 independent experiments. 
A

\begin{tabular}{|c|c|c|c|c|c|}
\hline Name & Sequence & Position(0-based) & Strand & Score & $p$-value \\
\hline RXRA::VDR(MA0074.1) & GGTTCTAAACGTTCA & 2802 & - & 7.88 & 0.00025 \\
\hline RXRA::VDR(MA0074.1) & ATGTCACCTAGTACC & 1508 & + & 7.35 & 0.0004 \\
\hline RXRA::VDR(MA0074.1) & AGGACAAAAGATACA & 2660 & + & 6.17 & 0.000825 \\
\hline RXRA::VDR(MA0074.1) & GGGATACAAAGTGCA & 2830 & - & 5.67 & 0.000925 \\
\hline RXRA::VDR(MA0074.1) & GGGGCATATAGTTTG & 3984 & - & 5.58 & 0.001 \\
\hline RXRA::VDR(MA0074.1) & GGGTTGCCAGGTTGC & 534 & - & 5.36 & 0.001125 \\
\hline RXRA::VDR(MA0074.1) & ATGTCACAGAGGTC & 43 & + & 5.16 & 0.0012 \\
\hline RXRA::VDR(MA0074.1) & GCGCCATCCAGTACA & 5422 & + & 5.1 & 0.001225 \\
\hline RXRA::VDR(MA0074.1) & GGGCAAAT GGATTCA & 4354 & - & 4.62 & 0.0016 \\
\hline RXRA::VDR(MA0074.1) & GGGTAAT GAGGTTT & 2087 & + & 4.47 & 0.001825 \\
\hline RXRA::VDR(MA0074.1) & AGTTCCTTGGGTAC & 3835 & - & 4.37 & 0.00195 \\
\hline RXRA::VDR(MA0074.1) & TGGTCCATAGGGTCA & 1755 & + & 4.3 & 0.001975 \\
\hline RXRA::VDR(MA0074.1) & GTCACCTAGTACC & 1510 & + & 3.48 & 0.003175 \\
\hline RXRA::VDR(MA0074.1) & AGTCCACAGCCTTCA & 5820 & + & 3.24 & 0.00375 \\
\hline RXRA::VDR(MA0074.1) & GTCTCAT GGGCTTCG & 485 & & 3.24 & 0.00375 \\
\hline
\end{tabular}

MYOGENIN PROMOTER (4kb upstream of START codon)

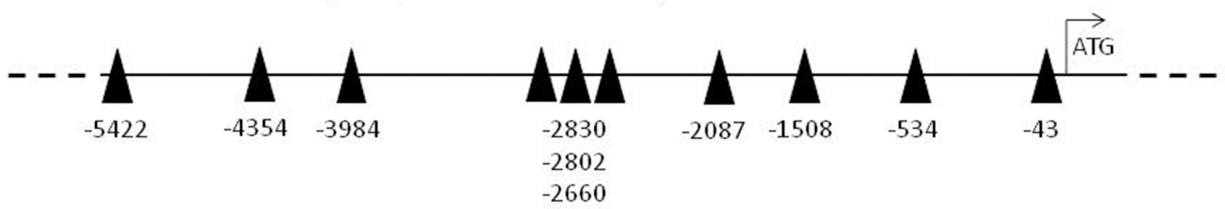

B
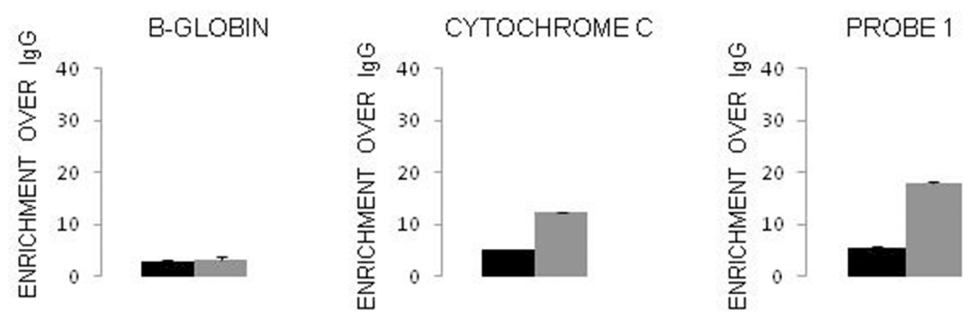

CONTROLS
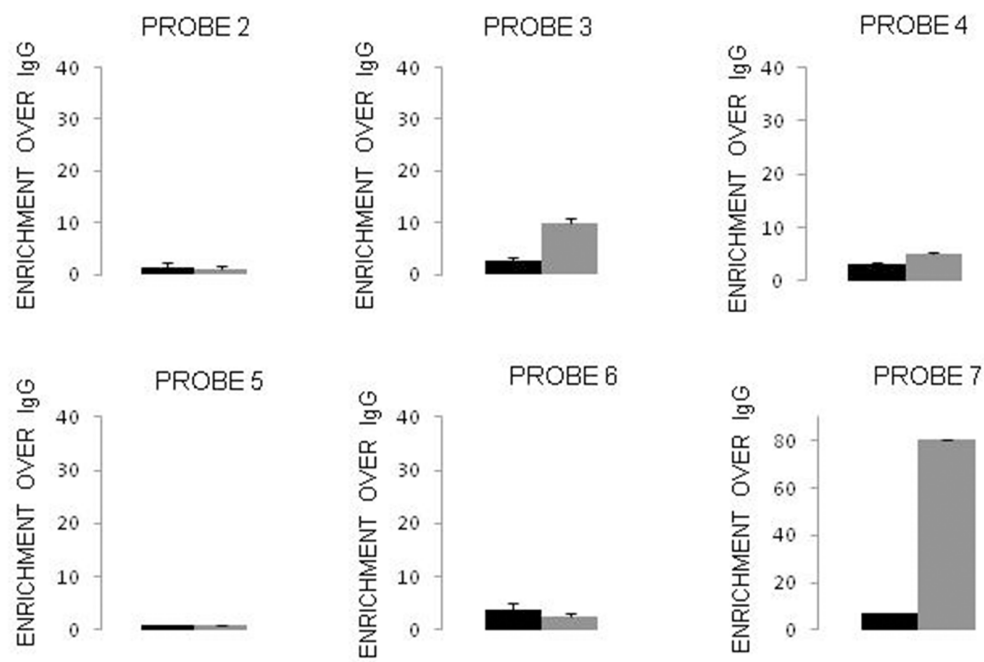

Figure 5: VDR is recruited at regulatory regions of the myogenin gene after VitD exposition. A. Putative VDR binding sites in the promoter region of the Myogenin gene $(6.5 \mathrm{Kbp})$, as predicted by LASAGNA-Search web tool, and schematic representation of the Myogenin promoter region showing putative binding sites for VDR; B. ChIP assay (representative pattern) was performed with chromatin extracted from $\mathrm{C} 2 \mathrm{C} 12$ myoblasts treated or untreated with VitD, using normal rabbit IgG and an anti-VDR antibody. $\beta$-globin and cytochrome c promoter regions were amplified as negative and positive controls, respectively. 
VitD3 (80 IU/kg/day) was administered to animals in which muscle injury has been induced by $\mathrm{BaCl}_{2}$ injection in the tibialis anterior muscle. As described in the literature, $\mathrm{BaCl}_{2}$ causes an extensive muscle injury and the subsequent regeneration involves about $90 \%$ of muscle fibers [29]. In untreated mice, total fiber CSA as well as the number of fiber with central nuclei, evaluated 15 days after damage, were not significantly different between injured and uninjured muscles, suggesting that complete regeneration had already occurred (Figures 7A, 7C). By contrast, and consistent with the in vitro results, in the damaged muscle, the CSA of VitD3-treated animals was decreased, while the number of fibers with central nuclei was higher than in the controlateral uninjured one (Figures 7A, 7C). In VitD3-supplemented mice VDR expression was markedly increased in the uninjured muscle, while it remained comparable to vehicle-treated animals in the regenerating one (Figure 7B and 7D). The expression level of myogenin and $\operatorname{Pax} 7$ were consistent with the impaired/delayed regeneration (Figure 7D). Finally, VitD3 administration proved unable to improve muscle wasting also in experimental arthritis (Supplementary Figure 4), a condition previously shown to be associated with enhanced muscle regeneration [30].

\section{DISCUSSION}

The present study is the first to investigate the involvement of VitD in the pathogenesis of cancer cachexia. Low VitD levels are a frequent feature in advanced cancer patients with cachexia and fatigue [31], while high serum VitD has been associated with low mortality in colorectal cancer patients [32]. However, data reported in the present study demonstrate that,

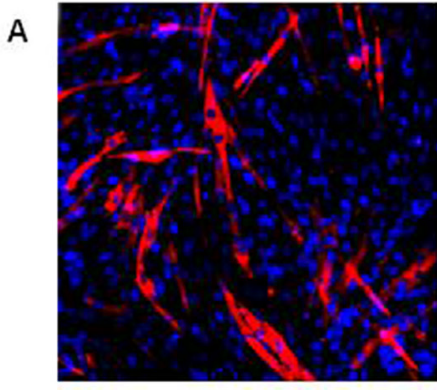

$\mathrm{ShC}$

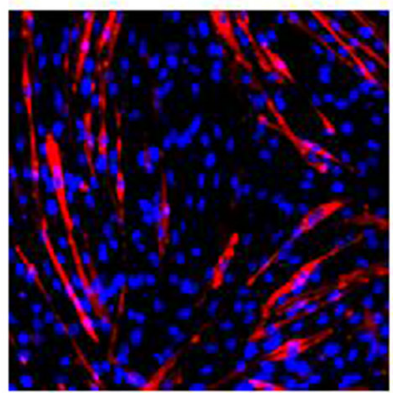

ShVDR

C

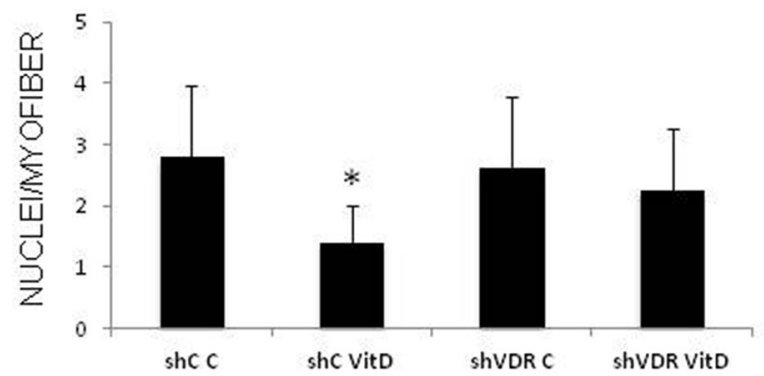

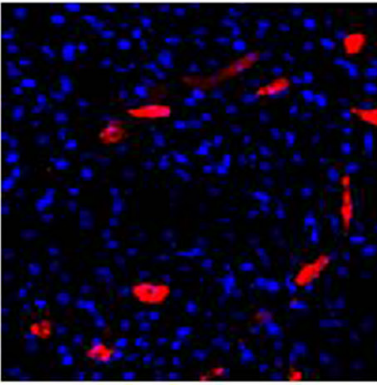

ShCVitD

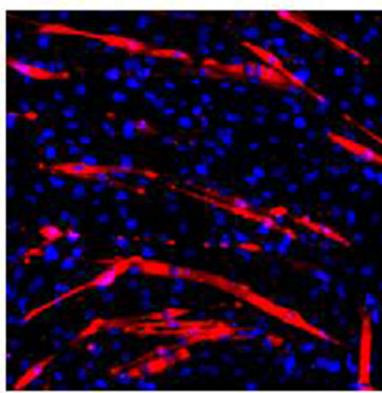

ShVDR VitD

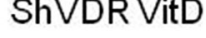

MYOG

TUB

B

MYHC

VDR
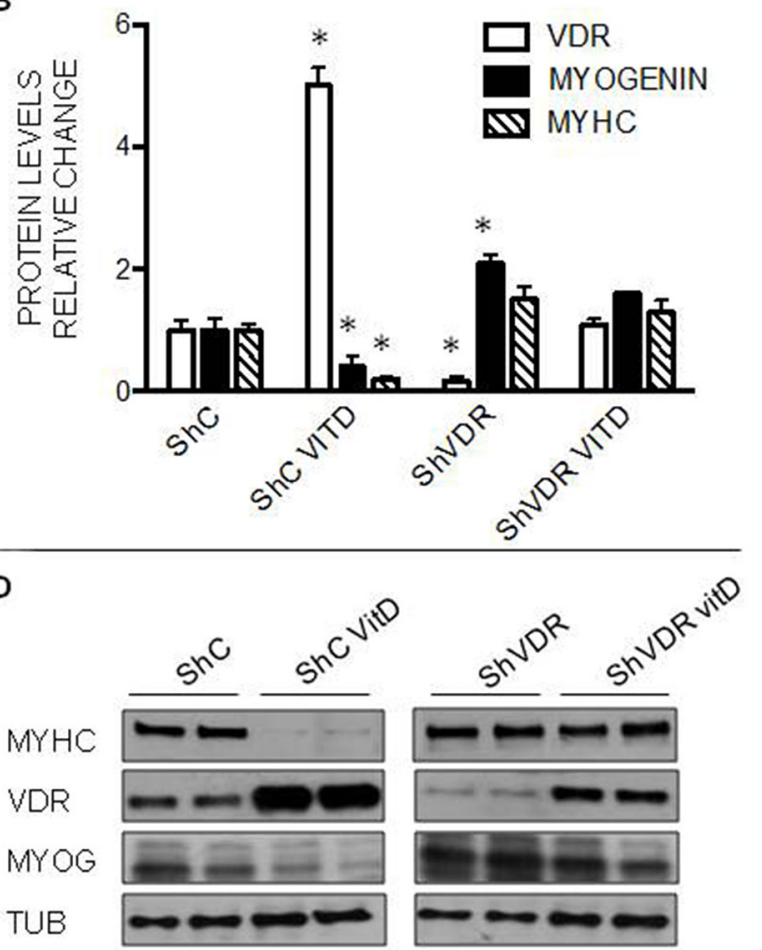

Figure 6: Effects of VitD treatment on VDR-silenced C2C12 cells. A. Immunostaining of C2C12 cells after 4 days of differentiation (Red: MyHC, Blue: nuclei). B. Protein expression levels of VDR, MyHC and myogenin at day 4 of differentiation. Data (means \pm SD) are expressed as relative change. C. number of nuclei/myofiber. D. representative western blotting pattern of MyHC, myogenin and VDR expression (Santa Cruz Biotechnology: anti-VDR antibody, clone D6, anti-myogenin antibody, clone F5D; Sigma: anti MyHC antibody, clone MY32). Significance of the differences: ${ }^{*} \mathrm{p}<0.05$ vs ShC, 2 independent experiments. 
depending on the experimental model of cancer cachexia chosen, circulating VitD levels may be reduced (AH130 hosts), unchanged (LLC hosts) or increased (C26 hosts) compared to control values. Irrespective of circulating VitD, however, the presence of the tumor was always associated with up-regulation of muscle VDR expression. This in vivo observation, coupled with the results obtained in vitro, suggests that VDR might play a role in the onset and/or progression of cachexia.

Quite recent observations show that VitD supplementation in patients with non-metastatic HER2 ${ }^{+}$ breast cancer treated with trastuzumab is associated with improved disease-free survival and with a tendency to increase body mass index [33]. Along this line, to assess if VitD supplementation could affect cancer cachexia, rats bearing the AH130 tumor, characterized by low VitD plasma levels, were treated with this hormone. The results show that body weight and muscle mass did not appreciably change in VitD-treated $v s$ untreated tumor hosts, although VitD plasma levels in the former were nearly restored to control values. Of interest, in the AH130 hosts treated with VitD, muscle VDR levels were higher than those detected in the untreated tumorbearing rats, likely in view of the restoration of normal circulating vitamin levels. In this regard, it is well known that VitD positively regulates the expression of its main receptor both in vivo and in vitro $[34,35]$. The other way round, the present study shows that increased VDR levels in the muscle of tumor-bearing animals seems a constant finding, irrespective of changes in circulating VitD levels. On the whole, these observations suggest that: i) a direct link between VitD plasma levels and the outcome of cancer cachexia is lacking; and ii) a ligandindependent dysregulation of VDR signaling pathway could be involved in the pathogenesis of cancer-induced muscle wasting. In this regard, in certain conditions VDR
A

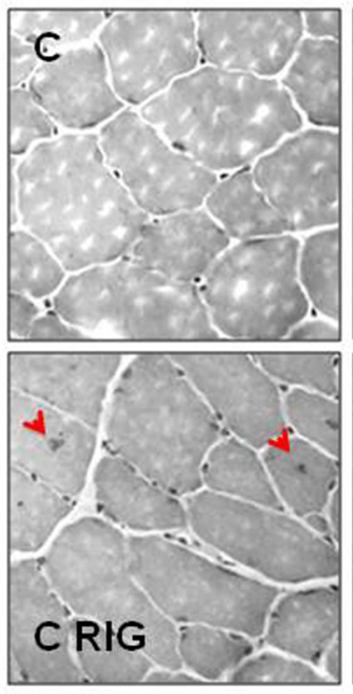

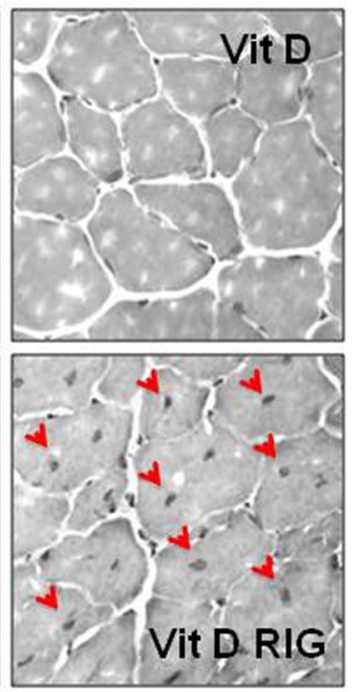

B

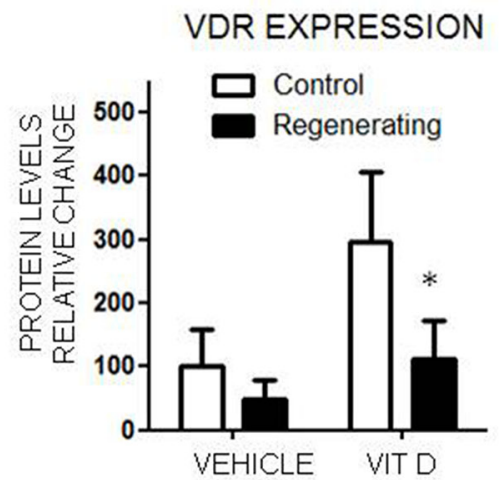

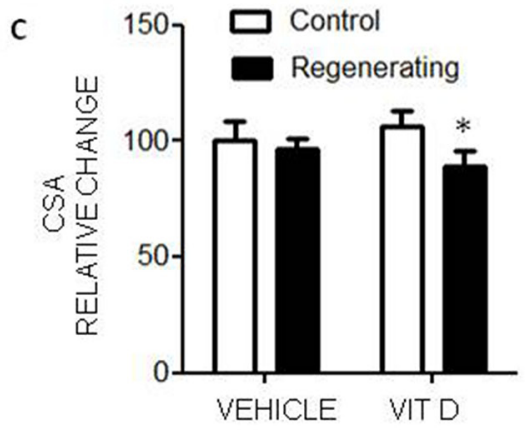

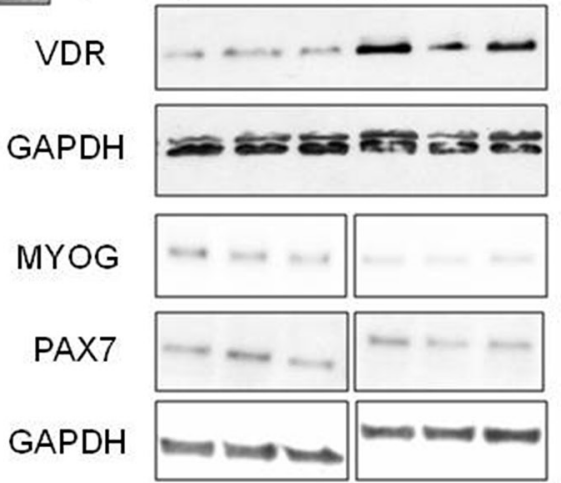

REGENERATING

\section{CONTROLS}

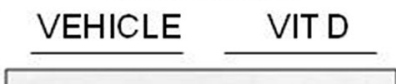

VEHICLE VIT D

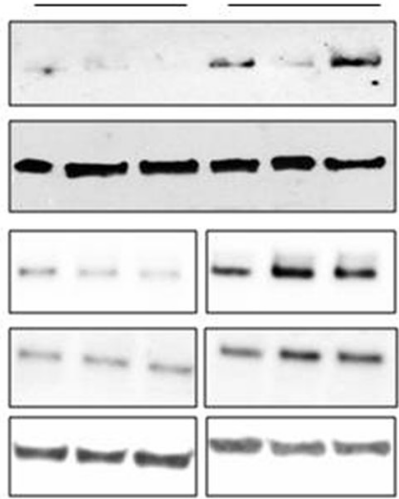

Figure 7: Effects of VitD administration during muscle regeneration. A. Histological pattern of regenerating and non regenerating muscles in VitD3-treated $(n=6)$ and untreated mice $(n=6)$. For experimental details see Materials and Methods. B. VDR expression in regenerating and non regenerating muscles in the presence or in the absence of VitD3 treatment (Santa Cruz Biotechnology: anti-VDR antibody, clone D6). Data (means \pm SD) are expressed as relative change. Significance of the differences: * $p<0.05$ vs C. C. Average myofiber CSA of controls (white bars) or regenerating (black bars) muscles 15 days after $\mathrm{BaCl}_{2}$ injection; 400 myofibers (regenerating or not)/condition have been counted. D. Representative samples showing VDR, Pax7 and myogenin expression (Santa Cruz Biotechnology: anti-VDR antibody, clone D6, anti-myogenin antibody, clone F5D; Developmental Studies Hybridoma Bank, University of Iowa: anti-Pax7 antibody). 
has been proposed to act independently of VitD, although it still requires functional VitD responsive elements as well as an intact VDR DNA binding domain; the effects deriving from such action are thus indistinguishable from VitD-dependent transactivation [36]. On this line, VDR up-regulation in the skeletal muscle of tumor hosts could be considered per se as a condition sufficient for its transcriptional activity. Consistent with such a mechanism, a VitD-independent, toll-like receptor (TLR)2-dependent VDR hyperactivation has been proposed to contribute to the regulation of innate immune response [37].

The observation that VDR is overexpressed in the skeletal muscle of tumor-bearing animals suggests that the VitD/VDR-dependent signaling pathway might contribute to cancer-induced muscle wasting, despite the lack of effect of VitD supplementation. In this regard, VDR relevance to skeletal muscle biology has been previously described. As an example, Endo and coworkers reported that VDR knock-out mice develop muscles smaller than those of wild-type animals, with high expression levels of myogenic regulatory factors (MRF) such as Myogenin, Myf5 and E2A. Moreover, the authors showed that MRF expression in myoblast cultures is decreased by VitD treatment. An opposite view is reported by a study showing that MRF expression is reduced in VitD deficient rats [14].

The results obtained in the present study on $\mathrm{C} 2 \mathrm{C} 12$ and primary myoblasts clearly show that VitD treatment impairs their differentiation into mature myotubes, likely due to the modulation of both VDR (increased) and myogenin (decreased) expression. In this regard, these observations are consistent with recent data by Girgis and coworkers showing that VitD inhibits myotube formation in $\mathrm{C} 2 \mathrm{C} 12$ cultures and that the few formed myotubes appeared bigger than those obtained in control C2C12 myocytes. The authors ascribed this latter effect to modulations of the myostatin-dependent pathway. No changes in myotube size were detected in the present study, however; the different experimental period (10 days in Girgis et al., 6 days in the present study) could account for such a discrepancy. The inhibition of myotube formation induced by vitD suggests an impairment of myogenesis. Consistent with this hypothesis, muscle regeneration after $\mathrm{BaCl}_{2}$-induced damage was altered in VitD-treated mice. The relevance of VDR to myogenic impairment in VitD-treated cells is demonstrated by the observation that normal differentiation was restored when VDR expression was silenced. Of interest, also in the absence of VitD stimulation, myogenin levels were higher in VDRsilenced than in wild-type $\mathrm{C} 2 \mathrm{C} 12$ cells, strengthening the hypothesis of a ligand-independent, VDR-mediated, negative regulation of myogenin transcription. Finally, the presence of several VDRE in the promoter region of the myogenin gene and the demonstration that VDR can directly bind the myogenin promoter support the proposed mechanism of regulation.
VDR expression in $\mathrm{C} 2 \mathrm{C} 12$ cells is high at the beginning of the differentiation process and is progressively reduced until the cells complete their maturation into myotubes. This observation is consistent with previous data reported in the literature, showing that mean intracellular VDR content is higher in undifferentiated than in differentiated cells [35, 38]. Moreover, VDR expression is sustained in myoblasts of different origins as well as in the developing skeletal muscle [21, 39, 40]. By contrast, VDR expression in the adult muscle or in differentiated myotubes is always reported as very low or even absent [41], although the results shown in the present study demonstrate that it can be stimulated by VitD treatment, while not by the proinflammatory cytokine IL-6. While the atrophyinducing properties of IL-6 are well described, little is known about the possibility that VitD, likely through VDR, can stimulate myonuclei loss and/or MyHC degradation in differentiated myotubes. In this regard, however, the data shown in the present study are more likely representative of an inhibition of still ongoing myonuclei accretion. Indeed, $\mathrm{C} 2 \mathrm{C} 12$ cultures are not completely differentiated after 4 days in differentiation medium (see Figure 3A). In addition, the suggestion that VitD does not stimulate protein degradation is supported by a previous report showing that protein synthesis induced by leucine and insulin in $\mathrm{C} 2 \mathrm{C} 12$ myotubes is enhanced in the presence of $10 \mathrm{nM}$ calcitriol [42].

Taken together, these considerations highlight that VDR-dependent signaling pathway is likely to participate in the regulation of the first phases of myogenic differentiation in vitro and of skeletal muscle development and regeneration in vivo. In this regard, VDR downregulation may represent a condition required to achieve complete myogenic differentiation. Such a hypothesis is supported by the results in this study showing that animals administered VitD display an impaired muscle regeneration that is associated with a tendency to increase VDR expression. These results are in agreement with a very recent study showing that a supraphysiological dose of VitD injected into damaged muscle (days 4-7 after $\mathrm{BaCl}_{2}$ ) delays the regenerative response [43]. The experimental setting shown in the present study, however, is quite different: the animals received VitD3, the administration route was intragastric, and treatment started the day of injury to finish the day before sacrifice.

An altered myogenic potential has been shown to contribute to cancer-induced muscle wasting $[5,6]$. Activation of the MAPK/ERK pathway plays a role in this regard, contributing to keep $\mathrm{Pax} 7^{+}$cells from proceeding along differentiation, leading to their accumulation into the skeletal muscle [6]. The results shown in the present study suggest that also VDR overexpression could contribute to the impaired myogenesis observed in tumor hosts, mainly by altering the expression of myogenic factors involved in the differentiation program, such as myogenin [6]. 
Interestingly, an interaction between ERK and VDR signaling pathways has previously been proposed: in MG63 (human osteoblastic osteosarcoma cell line) and HeLa (human cervical carcinoma cell line) cells, ERK activation leads to increased VDR transcriptional activity through overexpression of its coreceptor RXR gamma [44]. In this regard, ERK activation occurring in the skeletal muscle of cachectic tumor-bearing animals could be responsible for the establishment of a 'VDR-prone' environment, resulting in enhanced VDR-dependent signaling.

It is also worth citing that a relationship between VDR activation and skeletal muscle disorders has already been reported. The FokI polymorphism of VDR gene is a $\mathrm{T} / \mathrm{C}$ transition in the second exon, resulting in a truncated protein (424aa instead of 427aa) with enhanced transactivation capacity [45]. Two studies in humans suggest that FokI polymorphism is associated with decreased skeletal muscle mass and strength. In particular, Roth and coworkers showed that FokI homozygous men display a low fat-free muscle mass and a risk of sarcopenia 2.17-fold higher than controls [46]. The other study demonstrates that homozygosity for the FokI polymorphism is associated with reduced quadriceps strength compared with heterozygosity or control subjects [47]. Finally, a recent study on cancer patients shows a correlation between the presence of specific VDR BsmI and TaqI alleles and CRP plasma levels, one marker of cancer cachexia [48]. In particular, the fact that VDR $\mathrm{b}$ and $\mathrm{T}$ alleles are more frequent in cachectic cancer patients with elevated CRP levels leads to the conclusion that this may represent an early clinical predictor for more aggressive forms of cachexia [49]. Intriguingly, the VDR bT genotype has been previously demonstrated to be associated with high expression levels of both VDR mRNA and protein [45, 50-52].

In conclusion, the present study suggests that VDR overexpression in animal models of cancer cachexia is likely contributing to skeletal muscle wasting through an impairment of the muscle regenerative program. An important limitation of the present study is the lack of a vitamin D-depleted control. Indeed, most of the clinical trials reported in the literature describe the effects of vitamin $\mathrm{D}$ supplementation in a context of vitamin $\mathrm{D}$ deficiency, while at least in part the present data reflect the effects of supraphysiological vitamin D levels. Another point is that vitamin D-induced VDR overexpression in the AH130 hosts does not worsen muscle wasting, in some way opposing to the potential involvement of VDR in causing cancer-induced muscle depletion. However, muscle weight loss in the AH-130 hosts is rather severe, and the possibility that it cannot be further exacerbated should be taken into account.

On the basis of the results shown in the present study we can then postulate that a note of caution should be made when considering VitD supplementation in patients affected by cancer cachexia or any other condition of skeletal muscle atrophy where a regenerative process might be involved. In this regard, too much circulating VitD may cause a further increase in the expression levels of VDR that could decrease the regenerative potential of skeletal muscle precursor cells. Along this line, the decrease in VitD circulating levels observed in the AH130 hosts as well as in cancer patients [31] could represent a compensatory mechanism aimed at counteracting the increased VDR expression in the skeletal muscle. However, another possibility is that VitD plasma levels could be modulated independently from muscle VDR, for example by modulations of VitD-binding proteins. Finally, our in vitro experiments suggest that VDR is likely to play a fundamental role during myoblast differentiation, regulating and timing the expression of important myogenic factors, such as myogenin.

\section{MATERIALS AND METHODS}

\section{Reagents}

All reagents supplied by Sigma-Aldrich (St. Louis, MO, USA), unless differently specified.

\section{In vivo experiments}

Experimental animals were cared for in compliance with the Italian Ministry of Health Guidelines and the Policy on Humane Care and Use of Laboratory Animals (NIH, 1996). The experimental protocol was approved by the Bioethical Committee of the University of Torino (Torino, Italy). Male Wistar rats weighing approximately $150 \mathrm{~g}$, Balb-c and C57/BL6 mice weighing approximately $20 \mathrm{~g}$ were obtained from Charles River (Calco, Italy) and were maintained on a regular dark-light cycle (light from 8 a.m. to 8 p.m.), with free access to food and water during the whole experimental period. Rats were injected intraperitoneally with $10^{8}$ AH130 Yoshida ascites hepatoma cells [a gift many years ago from Prof. Ugo Del Monte (University of Milano, Milano, Italy) and maintained in our laboratory by weekly i.p. transplantation], whereas tumor-bearing mice $(n=8)$ were subcutaneously inoculated between the shoulder blades with $5 \times 10^{5}$ Colon 26 carcinoma cells [a gift from Prof. Mario P. Colombo (Istituto di Ricovero e Cura a Carattere Scientifico National Cancer Institute, Milano, Italy)] or with $5 \times 10^{5}$ Lewis Lung Carcinoma cells (ATCC, Manassas, VA). C26 and LLC cells were expanded to $50,000 / \mathrm{cm}^{2}$ in vitro in Dulbecco's modified Eagle's medium (DMEM) supplemented with 10\% fetal bovine serum, $100 \mathrm{U} / \mathrm{mL}$ penicillin, $100 \mu \mathrm{g} / \mathrm{mL}$ streptomycin, $100 \mu \mathrm{g} / \mathrm{mL}$ sodium pyruvate, and $2 \mathrm{mmol} / \mathrm{L}$ L-glutamine. Cells were maintained at $37^{\circ} \mathrm{C}$ in a humidified atmosphere of $5 \% \mathrm{CO}_{2}$ in air, detached with trypsin, resuspended in sterile saline, and subsequently implanted in the animals at the concentrations indicated above. Rats or mice 
inoculated with vehicle (saline) served as controls $(n=6)$. In the AH130 experiment, animals were randomized and divided into four groups: controls $(n=6)$ and AH130 $(n=$ 8), administered or not VitD3. Treated groups received intragastrically a daily administration of $80 \mathrm{IU} / \mathrm{kg}$ body weight (b.w.) of VitD3 dissolved in $200 \mu$ l of corn oil. Control rats were administered with an even amount of corn oil. The same treatment schedule was also applied to a group of mice, divided into controls (C) and C26 hosts (C26), treated or untreated with VitD3. VitD3 dosage was selected on the basis of data reported in the literature as well as by performing dose-response preliminary experiments in control animals, having VDR as endpoint. The intragastrical route of administration was chosen taking into consideration a potential translation to the clinical practice.

Animal weight and food intake were recorded daily. AH130-bearing rats, C26 hosts and LLC-bearing mice were euthanized under anesthesia 7, 14 and 28 days after tumor transplantation, respectively. Several muscles and organs were rapidly excised, weighed, frozen in isopentane cooled with liquid $\mathrm{N}_{2}$, and stored at $-80^{\circ} \mathrm{C}$.

In the regeneration experiment, skeletal muscle injury was induced in 8 week old mice by injecting $1.2 \% \mathrm{BaCl}_{2}(30$ $\mu \mathrm{l})$ into the left tibialis anterior muscle. The controlateral muscle was injected with PBS and used as control. Half of mice $(n=6)$ received VitD as described above. After 15 days of recovery the animals were euthanized and the tibialis muscles were dissected and removed, rapidly frozen in melting isopentane and used for cross-sectional area (CSA) and protein expression analysis.

Healthy or arthritic male Wistar rats $(150 \mathrm{~g} / 6$ weeks old) were purchased from Charles River. An intradermal injection of heat-inactivated Mycobacterium butyricum (4 mg) induced arthritis in the right paw of rats. Controls received just vehicle (paraffin oil, $100 \mu \mathrm{l}$ ). Animals were maintained on a regular dark-light cycle (light from 8 a.m. to 8 p.m.), with free access to food and water during the whole experimental period. Arthritic and control rats were divided into two groups: VitD treated (80 IU/kg b.w./day, intragastrical administration) or vehicle (corn oil) treated (sham). Body weight and arthritis severity were assessed daily. Evaluation of the latter was performed by measuring the arthritis index of each animal, which was scored by grading each paw from 0 to 4 , since inflammation of the paw is associated with radiological and histological alterations of the joints ([53]). After 15 days, rats were sacrificed and muscles (gastrocnemius, tibialis anterior and heart), liver and spleen were rapidly excised, weighed, frozen in liquid nitrogen, and stored at $-80^{\circ} \mathrm{C}$ for further analysis.

\section{Human samples}

Already available samples, deriving from a previous study [54], were used. Muscle biopsies were obtained from patients recruited at the M.G. Vannini Hospital in Rome (Italy) after signing an informed consent and after clearance by the local Ethical Committee. Briefly, patients undergoing abdominal surgery for cancer (mainly gastrointestinal tumors) or for non neoplastic reasons (used as controls) were included. The following exclusion criteria were adopted: liver failure, diabetes, metabolic acidosis, acute and chronic renal failure, sepsis, AIDS, inflammatory bowel diseases, acute and chronic hepatitis, autoimmune disorders and chronic obstructive pulmonary disease. Biopsy specimens were obtained during the initial phase of the operation from the rectus abdominis muscle (for details see [54]), immediately frozen in liquid nitrogen and stored at $-80^{\circ} \mathrm{C}$ until analysis.

\section{Vitamin D concentration}

For the determination of VitD circulating levels, blood samples were centrifuged at $600 \mathrm{~g}$ for $10 \mathrm{~min}$ and the separated plasma was stored at $-20^{\circ} \mathrm{C} .25 \mathrm{D}$ plasma levels were determined by radioimmunoassay (Immunodiagnostic Systems Holdings, Boldon, UK).

\section{Histological analysis}

Serial $10 \mu \mathrm{m}$-thick frozen sections were cut from cryopreserved tissue blocks, adhered to Superfrost Plus microscopy slides and stained with hematoxylin and eosin. All sections were examined by light microscopy (Nikon Eclipse TS100) and digital images were obtained with a Nikon COOLPIX 4500 camera. To assess myofiber CSA 400 fibers of gastrocnemius muscle sections were counted and measured using Image $\mathrm{J}$ software (http://rsb.info.nih. gov/ij/; NIH, Bethesda, MD).

\section{Cell cultures}

Murine $\mathrm{C} 2 \mathrm{C} 12$ skeletal myoblasts (ATCC) were grown in high-glucose DMEM supplemented with $10 \%$ fetal bovine serum, $100 \mathrm{U} / \mathrm{mL}$ penicillin, $100 \mu \mathrm{g} / \mathrm{mL}$ streptomycin, $100 \mu \mathrm{g} / \mathrm{mL}$ sodium pyruvate, and $2 \mathrm{mmol} / \mathrm{L}$ l-glutamine and maintained at $37^{\circ} \mathrm{C}$ in a humidified atmosphere of $5 \% \mathrm{CO}_{2}$ in air. Differentiation was induced by switching subconfluent cultures to DMEM supplemented with $2 \%$ horse serum (differentiation medium). In the differentiation experiments VitD (calcitriol; 1,25(OH)2 D dissolved in DMSO) was added to the medium at a final concentration of $10 \mathrm{nM}$ or 100 $\mathrm{nM}$, starting from the first day of differentiation. Since VitD is known to have a short half life at $37^{\circ} \mathrm{C}(5 / 8$ hours), the medium was replaced every day during the experiments. Control cells received an equal amount of DMSO.

\section{Gene silencing}

VDR silencing was achieved by using shRNA. A set of four MISSION shRNAs targeting VDR mRNA 
and cloned into plKO.1-puro expression vector was used. Third generation lentiviral particles (kindly provided by Prof. Ponzetto, CERMS - University of Torino, Torino, Italy) were used to deliver the shRNA to C2C12 cells. Cells were then incubated in their regular growth medium containing Puromycin $1 \mu \mathrm{g} / \mathrm{ml}$, in order to select for stably transformed cells. After testing the expression of VDR via western blotting analysis, cells infected with the shRNA providing the highest silencing were used for all the experiments (5'-CGGCCTGAGATCAATCACATTTAA CTCGAGTTAAATGTGATTGATCTCAGGTTTTT-3').

Control cells were infected with a plKO.1-puro plasmid containing a non-specific shRNA (MISSION pLKO.1puro Non-Mammalian shRNA Control Plasmid DNA).

\section{Western blotting analysis}

Approximately $50 \mathrm{mg}$ of gastrocnemius or tibialis muscle were homogenized in $80 \mathrm{mmol} / \mathrm{L}$ Tris- $\mathrm{HCl}, \mathrm{pH}$ 6.8, containing $100 \mathrm{mmol} / \mathrm{L}$ dithiothreitol, $70 \mathrm{mmol} / \mathrm{L}$ $\mathrm{SDS}$, and $1 \mathrm{mmol} / \mathrm{L}$ glycerol, with freshly added protease and phosphatase inhibitor cocktails; kept on ice for 30 minutes; centrifuged at $15,000 \times g$ for 10 minutes at $4^{\circ} \mathrm{C}$ and the supernatant collected. Protein concentration was assayed according to Bradford, using bovine serum albumin as working standard. $\mathrm{C} 2 \mathrm{C} 12$ cells were lysed on RIPA buffer $(50 \mathrm{mmol} / \mathrm{L}$ Tris- $\mathrm{HCl}, \mathrm{pH} 7.4,150 \mathrm{mmol} / \mathrm{L} \mathrm{NaCl}$, $1 \%$ Nonidet P-40, $0.25 \%$ sodium deoxycholate, $1 \mathrm{mmol} / \mathrm{L}$ phenylmethylsulfonyl fluoride) with freshly added protease and phosphatase inhibitor cocktails. Equal amounts of protein $(30 \mu \mathrm{g})$ were heat-denaturated in sample-loading buffer (50 $\mathrm{mmol} / \mathrm{L}$ Tris- $\mathrm{HCl}, \mathrm{pH} 6.8,100 \mathrm{mmol} / \mathrm{L}$ dithiothreitol, 2\% SDS, $0.1 \%$ bromophenol blue, $10 \%$ glycerol), resolved by SDS-PAGE, and transferred to nitrocellulose membranes (Bio-Rad Laboratories, Hercules, CA). The filters were blocked with Tris-buffered saline containing $0.05 \%$ Tween and 5\% nonfat dry milk and then were incubated overnight with antibodies directed against VDR (mouse monoclonal antibody, clone D6, Santa Cruz Biotechnology, Santa Cruz, CA), Myogenin (mouse monoclonal antibody, clone F5D, Santa Cruz Biotechnology, Santa Cruz, CA), MyHC (mouse monoclonal antibody, clone MY32), Pax7 (Developmental Studies Hybridoma Bank, University of Iowa), MyoD (rabbit polyclonal antibody, clone M318, Santa Cruz Biotechnology, Santa Cruz, CA), GAPDH (goat polyclonal antibody, V18, Santa Cruz Biotechnology, Santa Cruz, CA) and tubulin (mouse monoclonal antibody, clone T5168). Peroxidaseconjugated IgGs (Bio-Rad Laboratories) were used as secondary antibodies. Quantification of the bands was performed by densitometric analysis (TotalLab; Nonlinear Dynamics, Newcastle on Tyne, UK).

\section{Real time PCR}

Total RNA was obtained using TriPure isolation reagent (Roche Applied Science, Indianapolis, IN) following the manufacturer's instructions. RNA concentration was determined fluorometrically using RiboGreen reagent (Invitrogen, Carlsbad, CA). RNA integrity was checked by electrophoresis on $1.2 \%$ agarose gel containing $0.02 \mathrm{~mol} / \mathrm{L}$ morpholinopropanesulfonic acid and $18 \%$ formaldehyde. Total mRNA was retrotranscribed using an iScript cDNA synthesis kit (BioRad Laboratories). Transcript levels were determined by real-time PCR using the SsoFast EvaGreen supermix and the MiniOpticon thermal cycler (Bio-Rad Laboratories), normalizing the expression for TBP. Primer sequences (Invitrogen, Carlsbad, CA) were as follows:

VDR FW CCTCATAAAGTTCCAGGTGGGG

VDR RV GGATAGGCGGTCCTGAATGG

TBP FW TGTCCAGAGCACCAACAGTC

TBP RV TAACAGCAGCAAAACGCTTG

\section{Promoter analysis and chromatin immunoprecipitation assay (ChIP)}

The promoter region (-6000bp, +500bp) of murine myogenin gene was analyzed for the presence of putative VDR binding sites (Matrix-Derived JASPAR CORE Models, Vertebrates, ID: MA0074.1) using the LASAGNA-Search web tool [55].

Chromatin was isolated from $\mathrm{C} 2 \mathrm{C} 12$ myoblast untreated or treated during 4 hours with VitD in DMSO at a final concentration of $100 \mathrm{nM}$. ChIP was performed according to [56]. Cells were cross-linked adding to tissue culture medium PBS and 1\% formaldehyde and incubating for 10 minutes. Crosslinking was blocked with $0.125 \mathrm{M}$ glycine for 5 minutes. Cells were scraped and subsequently lysed in RIPA buffer (see above). Nuclei were pelleted by microcentrifugation and lysed by incubation in nuclear lysis buffer (1\% sodium dodecyl sulfate, 10 mM EDTA, 50 $\mathrm{mM}$ Tris-chloride $\mathrm{pH} 8.1,0.5 \mathrm{mM}$ phenylmethylsulfonyl fluoride, protease inhibitors). The resulting chromatin solution was sonicated to obtain fragments of approximately $0.5 \mathrm{~kb}$ and immunoprecipitated with 3.5 $\mu \mathrm{g}$ of rabbit $\mathrm{IgG}$ or VDR (rabbit polyclonal antibody, clone C-20; Santa Cruz Biotechnology, Santa Cruz, CA). Immunoprecipitation procedures were performed according to [57]. Quantitative Real Time PCR was performed using SYBR green IQ reagent (Bio-Rad Laboratories) with CFX Connect detection system (BioRad Laboratories). Primer sequences:

$\beta$-globin

FW GACAAACATTATTCAGAGGGAGT

RV AAGCAAATGTGAGGAGCAACTGAT

Cytochrome c

FW GGAGGCAAGCATAAGACTGG

RV TCCATCAGGGTATCCTCTCC

Probe 1

FW TGCAATGTCACAGAGGTCTAAGC RV CTACACAGAAGGAGACAGAGGCTC Probe 2 
FW CTCCCTCTTCCTTCTCCTTCAGC

RV CAACCCAGGTCAGCCTATCAGTA

Probe 3

FW CACACAGGGTAGCAGGTAGATGAC

RV CACTATCATACCTTGCTTACCCAC

Probe 4

FW CAGGAACCTGCAAGGCATCAAAG

RV GTCATACCTGCTGTGGAAACTGC

Probe 5

FW AAGGAGGAAACAGGTGTGTGAGG

RV GCTGCACTTTGTATCCCC

Probe 6

FW CGTTCAACACACTTTCCACCTCC

RV GCAGTTTCCACAGCAGGTATGAC

Probe 7

FW TAGTCGGCCATCATTGGGAAGAG

RV CCTACCCACTCATTCCCACTTCT

\section{Immunofluorescence}

C2C12 or primary cells were washed with PBS and fixed in 1:1 acetone:methanol. Samples were then probed with the following primary antibodies: MyHC (mouse monoclonal antibody, clone MY32), VDR (mouse monoclonal antibody, clone D6. Santa Cruz Biotechnology, Santa Cruz, CA), Myogenin (mouse monoclonal antibody, clone F5D. Santa Cruz Biotechnology, Santa Cruz, CA). Detection was performed using Alexa Fluor secondary antibodies (Life Technologies, Grand Island, NY). Nuclei were stained with DAPI fluorochrome, and the images were captured using an epi-illuminated fluorescence microscope (Axiovert 35; Carl Zeiss MicroImaging $\mathrm{GmbH}$, Jena, Germany). About 200 myotubes/conditions were analyzed to assess the number of nuclei and myotube diameter.

\section{Statistical analysis}

All the results are expressed as means $\pm \mathrm{SD}$, except for Real Time PCR gene expression (means \pm SEM). Representative Western blots show independent samples. The significance of the differences was evaluated by ANOVA followed by Tukey's test.

\section{CONFLICTS OF INTEREST}

The authors have no conflict of interest to declare.

\section{GRANT SUPPORT}

Work supported by Associazione Italiana per la Ricerca sul Cancro (AIRC - P.C.: IG9153), Milano, Ministero per l'Università e la Ricerca, Roma (PRIN projects), University of Torino (ex-60\% funds), Regione Piemonte, Italy.

\section{REFERENCES}

1. Muscaritoli M, Molfino A, Lucia S and Rossi Fanelli F. Cachexia: a preventable comorbidity of cancer. A T.A.R.G.E.T. approach. Crit Rev Oncol Hematol. 2015; 94:251-259.

2. Argiles JM, Busquets S, Stemmler B and Lopez-Soriano FJ. Cancer cachexia: understanding the molecular basis. Nat Rev Cancer. 2014; 14:754-762.

3. Suzuki H, Asakawa A, Amitani H, Nakamura N and Inui A. Cancer cachexia--pathophysiology and management. J Gastroenterol. 2013; 48:574-594.

4. Baracos VE, DeVivo C, Hoyle DH and Goldberg AL. Activation of the ATP-ubiquitin-proteasome pathway in skeletal muscle of cachectic rats bearing a hepatoma. Am J Physiol. 1995; 268:E996-1006.

5. He WA, Berardi E, Cardillo VM, Acharyya S, Aulino P, Thomas-Ahner J, Wang J, Bloomston M, Muscarella P, Nau P, Shah N, Butchbach ME, Ladner K, et al. NF-kappaB-mediated Pax7 dysregulation in the muscle microenvironment promotes ancer cachexia. J Clin Invest. 2013; 123:4821-4835.

6. Penna F, Costamagna D, Fanzani A, Bonelli G, Baccino FM and Costelli P. Muscle wasting and impaired myogenesis in tumor bearing mice are prevented by ERK inhibition. PLoS One. 2010; 5:e13604.

7. Omdahl JL, Morris HA and May BK. Hydroxylase enzymes of the vitamin D pathway: expression, function, and regulation. Annu Rev Nutr. 2002; 22:139-166.

8. Rosen CJ, Adams JS, Bikle DD, Black DM, Demay MB, Manson JE, Murad MH and Kovacs CS. The nonskeletal effects of vitamin D: an Endocrine Society scientific statement. Endocr Rev. 2012; 33:456-492.

9. Li YC, Pirro AE, Amling M, Delling G, Baron R, Bronson $\mathrm{R}$ and Demay MB. Targeted ablation of the vitamin D receptor: an animal model of vitamin D-dependent rickets type II with alopecia. Proc Natl Acad Sci U S A. 1997; 94:9831-9835.

10. Zhao $G$ and Simpson RU. Membrane localization, Caveolin-3 association and rapid actions of vitamin D receptor in cardiac myocytes. Steroids. 2010; 75:555-559.

11. Garabedian M, Tanaka Y, Holick MF and Deluca HF. Response of intestinal calcium transport and bone calcium mobilization to 1,25-dihydroxyvitamin D3 in thyroparathyroidectomized rats. Endocrinology. 1974; 94:1022-1027.

12. Zittermann A. Vitamin D in preventive medicine: are we ignoring the evidence? Br J Nutr. 2003; 89:552-572.

13. Wong YY, McCaul KA, Yeap BB, Hankey GJ and Flicker L. Low vitamin D status is an independent predictor of increased frailty and all-cause mortality in older men: the Health in Men Study. J Clin Endocrinol Metab. 2013; 98:3821-3828. 
14. Bhat M, Kalam R, Qadri SS, Madabushi S and Ismail A. Vitamin D deficiency-induced muscle wasting occurs through the ubiquitin proteasome pathway and is partially corrected by calcium in male rats. Endocrinology. 2013; 154:4018-4029.

15. Moreira-Pfrimer LD, Pedrosa MA, Teixeira L and LazarettiCastro M. Treatment of vitamin D deficiency increases lower limb muscle strength in institutionalized older people independently of regular physical activity: a randomized double-blind controlled trial. Ann Nutr Metab. 2009; 54:291-300.

16. Bischoff-Ferrari HA, Dawson-Hughes B, Staehelin HB, Orav JE, Stuck AE, Theiler R, Wong JB, Egli A, Kiel DP and Henschkowski J. Fall prevention with supplemental and active forms of vitamin D: a meta-analysis of randomised controlled trials. BMJ. 2009; 339:b3692.

17. Broe KE, Chen TC, Weinberg J, Bischoff-Ferrari HA, Holick MF and Kiel DP. A higher dose of vitamin d reduces the risk of falls in nursing home residents: a randomized, multiple-dose study. J Am Geriatr Soc. 2007; 55:234-239.

18. Lips P, Graafmans WC, Ooms ME, Bezemer PD and Bouter LM. Vitamin D supplementation and fracture incidence in elderly persons. A randomized, placebo-controlled clinical trial. Ann Intern Med. 1996; 124:400-406.

19. Annweiler C, Beauchet O, Berrut G, Fantino B, Bonnefoy M, Herrmann FR and Schott AM. Is there an association between serum 25-hydroxyvitamin D concentration and muscle strength among older women? Results from baseline assessment of the EPIDOS study. J Nutr Health Aging. 2009; 13:90-95.

20. Boland R. Role of vitamin D in skeletal muscle function. Endocr Rev. 1986; 7:434-448.

21. Endo I, Inoue D, Mitsui T, Umaki Y, Akaike M, Yoshizawa T, Kato $\mathrm{S}$ and Matsumoto T. Deletion of vitamin D receptor gene in mice results in abnormal skeletal muscle development with deregulated expression of myoregulatory transcription factors. Endocrinology. 2003; 144:5138-5144.

22. Costelli P, Carbo N, Tessitore L, Bagby GJ, Lopez-Soriano FJ, Argiles JM and Baccino FM. Tumor necrosis factoralpha mediates changes in tissue protein turnover in a rat cancer cachexia model. J Clin Invest. 1993; 92:2783-2789.

23. Okuno H, Kishimoto KN, Hatori M and Itoi E. 1alpha,25dihydroxyvitamin $\mathrm{D}(3)$ enhances fast-myosin heavy chain expression in differentiated $\mathrm{C} 2 \mathrm{C} 12$ myoblasts. Cell Biol Int. 2012; 36:441-447.

24. Girgis CM, Clifton-Bligh RJ, Mokbel N, Cheng K and Gunton JE. Vitamin D signaling regulates proliferation, differentiation, and myotube size in $\mathrm{C} 2 \mathrm{C} 12$ skeletal muscle cells. Endocrinology. 2014; 155:347-357.

25. Penna F, Busquets S, Pin F, Toledo M, Baccino FM, Lopez-Soriano FJ, Costelli P and Argiles JM. Combined approach to counteract experimental cancer cachexia: eicosapentaenoic acid and training exercise. J Cachexia Sarcopenia Muscle. 2011; 2:95-104.
26. Gorman S, Scott NM, Tan DH, Weeden CE, Tuckey RC, Bisley JL, Grimbaldeston MA and Hart PH. Acute erythemal ultraviolet radiation causes systemic immunosuppression in the absence of increased 25-hydroxyvitamin D3 levels in male mice. PLoS One. 2012; 7:e46006.

27. Irazoqui AP, Boland RL and Buitrago CG. Actions of 1,25(OH)2-vitamin D3 on the cellular cycle depend on VDR and p38 MAPK in skeletal muscle cells. J Mol Endocrinol. 2014; 53:331-343.

28. Bonetto A, Aydogdu T, Jin X, Zhang Z, Zhan R, Puzis L, Koniaris LG and Zimmers TA. JAK/STAT3 pathway inhibition blocks skeletal muscle wasting downstream of IL-6 and in experimental cancer cachexia. Am J Physiol Endocrinol Metab. 2012; 303:E410-421.

29. Casar JC1 C-VC, Olguin H, Aldunate R, Inestrosa NC, Brandan E. Heparan sulfate proteoglycans are increased during skeletal muscle regeneration: requirement of syndecan-3 for successful fiber formation. J Cell Sci. 2004; 117:73.84.

30. de Oliveira Nunes Teixeira V, Filippin LI, Viacava PR, de Oliveira PG and Xavier RM. Muscle wasting in collageninduced arthritis and disuse atrophy. Exp Biol Med (Maywood). 2013; 238:1421-1430.

31. Dev R, Del Fabbro E, Schwartz GG, Hui D, Palla SL, Gutierrez N and Bruera E. Preliminary report: vitamin D deficiency in advanced cancer patients with symptoms of fatigue or anorexia. Oncologist. 2011; 16:1637-1641.

32. Mohr SB, Gorham ED, Kim J, Hofflich H, Cuomo RE and Garland CF. Could vitamin D sufficiency improve the survival of colorectal cancer patients? J Steroid Biochem Mol Biol. 2015; 148:239-244.

33. Zeichner SB, Koru-Sengul T, Shah N, Liu Q, Markward NJ, Montero AJ, Gluck S, Silva O and Ahn ER. Improved clinical outcomes associated with vitamin D supplementation during adjuvant chemotherapy in patients with HER2+ nonmetastatic breast cancer. Clin Breast Cancer. 2015; 15:e1-11.

34. Favus MJ, Mangelsdorf DJ, Tembe V, Coe BJ and Haussler MR. Evidence for in vivo upregulation of the intestinal vitamin $\mathrm{D}$ receptor during dietary calcium restriction in the rat. J Clin Invest. 1988; 82:218-224.

35. Solvsten H, Svendsen ML, Fogh K and Kragballe K. Upregulation of vitamin D receptor levels by $1,25(\mathrm{OH}) 2$ vitamin D3 in cultured human keratinocytes. Arch Dermatol Res. 1997; 289:367-372.

36. Ellison TI, Eckert RL and MacDonald PN. Evidence for 1,25-dihydroxyvitamin D3-independent transactivation by the vitamin D receptor: uncoupling the receptor and ligand in keratinocytes. J Biol Chem. 2007; 282:10953-10962.

37. Liu PT, Schenk M, Walker VP, Dempsey PW, Kanchanapoomi M, Wheelwright M, Vazirnia A, Zhang X, Steinmeyer A, Zugel U, Hollis BW, Cheng G and Modlin RL. Convergence of IL-1beta and VDR activation pathways in human TLR2/1induced antimicrobial responses. PLoS One. 2009; 4:e5810. 
38. Kong $\mathrm{J}$ and Li YC. Molecular mechanism of 1,25-dihydroxyvitamin D3 inhibition of adipogenesis in 3T3-L1 cells. Am J Physiol Endocrinol Metab. 2006; 290:E916-924.

39. Boland R, Norman A, Ritz E and Hasselbach W. Presence of a 1,25-dihydroxy-vitamin D3 receptor in chick skeletal muscle myoblasts. Biochem Biophys Res Commun. 1985; 128:305-311.

40. Buitrago $\mathrm{C}$ and Boland R. Caveolae and caveolin-1 are implicated in 1alpha,25(OH)2-vitamin D3-dependent modulation of Src, MAPK cascades and VDR localization in skeletal muscle cells. J Steroid Biochem Mol Biol. 2010; 121:169-175.

41. Wang Y and DeLuca HF. Is the vitamin $d$ receptor found in muscle? Endocrinology. 2011; 152:354-363.

42. Salles J1 CA, Giraudet C, Patrac V, Pierre P, Jourdan M, Luiking YC, Verlaan S, Migné C, Boirie Y, Walrand S. 1,25(OH)2-vitamin D3 enhances the stimulating effect of leucine and insulin on protein synthesis rate through $\mathrm{Akt} / \mathrm{PKB}$ and mTOR mediated pathways in murine C2C12 skeletal myotubes. Mol Nutr Food Res. 2013; 57:2137-2146.

43. Srikuea R HM. Effects of intramuscular administration of $1 \alpha, 25(\mathrm{OH}) 2 \mathrm{D} 3$ during skeletal muscle regeneration on regenerative capacity, muscular fibrosis, and angiogenesis. J Appl Physiol (1985). 2016; 120:1381-1393.

44. Narayanan R, Sepulveda VA, Falzon M and Weigel NL. The functional consequences of cross-talk between the vitamin D receptor and ERK signaling pathways are cell-specific. J Biol Chem. 2004; 279:47298-47310.

45. Whitfield GK, Remus LS, Jurutka PW, Zitzer H, Oza AK, Dang HT, Haussler CA, Galligan MA, Thatcher ML, Encinas Dominguez C and Haussler MR. Functionally relevant polymorphisms in the human nuclear vitamin $D$ receptor gene. Mol Cell Endocrinol. 2001; 177:145-159.

46. Roth SM, Zmuda JM, Cauley JA, Shea PR and Ferrell RE. Vitamin D receptor genotype is associated with fat-free mass and sarcopenia in elderly men. J Gerontol A Biol Sci Med Sci. 2004; 59:10-15.

47. Hopkinson NS, Li KW, Kehoe A, Humphries SE, Roughton M, Moxham J, Montgomery H and Polkey MI. Vitamin D receptor genotypes influence quadriceps strength in chronic obstructive pulmonary disease. Am J Clin Nutr. 2008; 87:385-390.
48. Barber MD, Ross JA and Fearon KC. Changes in nutritional, functional, and inflammatory markers in advanced pancreatic cancer. Nutr Cancer. 1999; 35:106-110.

49. Punzi T, Fabris A, Morucci G, Biagioni P, Gulisano M, Ruggiero M and Pacini S. C-reactive protein levels and vitamin $\mathrm{d}$ receptor polymorphisms as markers in predicting cachectic syndrome in cancer patients. Mol Diagn Ther. 2012; 16:115-124.

50. Luo XY, Yang MH, Wu FX, Wu LJ, Chen L, Tang Z, Liu NT, Zeng XF, Guan JL and Yuan GH. Vitamin D receptor gene BsmI polymorphism B allele, but not BB genotype, is associated with systemic lupus erythematosus in a Han Chinese population. Lupus. 2012; 21:53-59.

51. Ogunkolade BW, Boucher BJ, Prahl JM, Bustin SA, Burrin JM, Noonan K, North BV, Mannan N, McDermott MF, DeLuca HF and Hitman GA. Vitamin D receptor (VDR) mRNA and VDR protein levels in relation to vitamin D status, insulin secretory capacity, and VDR genotype in Bangladeshi Asians. Diabetes. 2002; 51:2294-2300.

52. Verbeek W, Gombart AF, Shiohara M, Campbell M and Koeffler HP. Vitamin D receptor: no evidence for allelespecific mRNA stability in cells which are heterozygous for the Taq I restriction enzyme polymorphism. Biochem Biophys Res Commun. 1997; 238:77-80.

53. Guglielmotti A, D'Onofrio E, Coletta I, Aquilini L, Milanese $\mathrm{C}$ and Pinza M. Amelioration of rat adjuvant arthritis by therapeutic treatment with bindarit, an inhibitor of MCP-1 and TNF-alpha production. Inflamm Res. 2002; 51:252-258.

54. Aversa Z PF, Lucia S, Penna F, Verzaro R, Fazi M, Colasante G, Tirone A, Rossi Fanelli F, Ramaccini C, Costelli P, Muscaritoli M. Autophagy is induced in the skeletal muscle of cachectic cancer patients. Sci Rep. 2016; 6:30340.

55. Lee C HC. LASAGNA-Search: an integrated web tool for transcription factor binding site search and visualization. Biotechniques. 2013; 54:141-153.

56. Savic D GJ, Jain P, Cooper GM, Myers RM. Mapping genome-wide transcription factor binding sites in frozen tissues. Epigenetics Chromatin. 2013; 6:30.

57. Proserpio V FR, Ryall JG, Sartorelli V, Caretti G. The methyltransferase SMYD3 mediates the recruitment of transcriptional cofactors at the myostatin and c-Met genes and regulates skeletal muscle atrophy. Genes Dev. 2013; 27:1299-1312. 